\title{
IRON OXIDE AND SODIUM HYDROXIDE ELIMINATION OF HEAVY METAL TOXICITY IN WHEAT SEEDLINGS
}

\author{
QIAO, L.* - LIU, H. - HU, C. - HU, L. - DU, H. \\ College of Life Science and Agronomy, Zhoukou Normal University, Zhoukou 466001, China \\ *Corresponding author \\ e-mail:qiaolin2012@126.com
}

(Received $15^{\text {th }}$ Sep 2018; accepted $12^{\text {th }}$ Nov 2018)

\begin{abstract}
Wheat is an important food crop in China. Iron oxide has a toxic effect on the growth and development of wheat. In this paper, wheat was used as experimental raw material to study the toxicological mechanism of $\mathrm{Cr}$ salt on wheat seed germination. To study the effects of hydrogen sulfide $\left(\mathrm{H}_{2} \mathrm{~S}\right)$ on the germination and seedling growth of wheat under Iron oxide salt stress, $\mathrm{H}_{2} \mathrm{~S}$ donor sodium hydroxide pretreatment could alleviate the inhibition of Iron oxide stress on wheat seed germination and seedling growth could promote the growth of wheat seed germ. It did not significantly promote the growth of radicle. Relief mechanism of sodium sulfide and hydrogen sulfide donor on the growth toxicity of wheat seedlings under Iron oxide salt stress was conducted. The optimum concentration of sodium hydroxide pretreatment was $0.08 \mathrm{mml} / \mathrm{L}$. At the same time, sodium hydroxide pretreatment reduced the content of superoxide anion and Malondialdehyde (MDA), alleviated the damage of wheat seedlings, and enhanced the stress resistance of wheat seedlings under Iron oxide stress. In summary, $\mathrm{H}_{2} \mathrm{~S}$ as a signal molecule can mediate the antioxidant response of crop seed germination to Iron oxide stress and can relieve Iron oxide stress-induced oxidative damage to crops. It also can enhance crop resistance under specific circumstances.
\end{abstract}

Keywords: Cr salt, sodium hydroxide, heavy metal, toxic mechanism, wheat seeds germination

\section{Introduction}

Almost all green plants on the Earth start from the seed germination, so the germination of seeds plays a crucial role in plant growth and development. Seed plants are usually divided into gymnosperms and angiosperms. Most of the angiosperm seed structure is basically the same, including seed coat, endosperm and embryo in three parts (Aoyama and Okamura, 2010). The seed coat is the outer shell that surrounds the seed and is in the outermost layer of the seed, which protects the embryo and endosperm of the seed pair. The endosperm is an important structure for the storage of nutrients inside the seeds. Before the seeds can be used for photosynthesis, the energy and substances needed for the seed germination process come from the conversion of the energy substances in the endosperm. In some seeds without endosperm, nutrients of the seeds are stored in the cotyledons (Schulze, 1984). The embryo is the most important part of the entire structure of the seed and can be seen as a prototype of the plant before it matures (Awad, 2000; Bauer and Blodau, 2006).

In some researches (Bose and Bhattacharyya, 2008; Bowell, 1994), the toxicological effects of four heavy metals $(\mathrm{Cu}, \mathrm{Cd}, \mathrm{Pb}$ and $\mathrm{Zn})$ in soil on wheat seedlings and the dynamic enrichment of heavy metals under the interaction of single and two were systematically studied by dish culture and pot experiments. The micro-damage of heavy metals to wheat seedlings was studied by transmission electron microscopy, and the stress of heavy metals was also discussed. The effects of heavy metal interaction on the physiological characteristics of wheat seedlings and the enrichment characteristics of heavy metals in wheat seedlings were systematically studied in Cenci and Morozzi's 
research (Cenci and Morozzi, 1977). The results showed that the interaction had antagonistic effects on wheat growth and physiological indices in varying degrees, among which root elongation was the most important (coefficient of variation was the largest). The effects of elements and their interaction on the enrichment of $\mathrm{Cu}, \mathrm{Cd}$ and $\mathrm{Zn}$ in the interaction combination were above significant level, while the effects on the enrichment of $\mathrm{Pb}$ were all very significant level. The enrichment characteristics of heavy metals in wheat plants in different growth periods were systematically analyzed by orthogonal experiment in Hanesch's paper (Hanesch, 2010) and the results showed that the enrichment amount of heavy metals in roots, stems and leaves was greater than that in glume shells and seeds. The result also showed the enrichment amount was significantly correlated with heavy metal stress. Under the interaction of heavy metals, different parts of wheat in different growth stages have different characteristics of heavy metal enrichment. Most of the heavy metal accumulation in different parts of wheat was significantly correlated with the stress of the metal, and the correlation decreased with the growth of wheat.

Oxidative damage destroys the normal physiological environment of the plant. Pretreatment can inhibit the decrease of endogenous content to a certain extent, provide a relatively reducing environment, relieve excessive oxidative environment, and increase the stress resistance of the plant. It can also effectively inhibit the increase of lipid membrane permeability, protect the integrity of lipid membrane, and reduce the induced plasma membrane oxidative damage (Mishra and Gopal, 2008). Treatment can inhibit the decrease of chlorophyll content to a certain extent. The treatment reduced the content of reactive oxygen species and reduced oxidative damage by inducing the increase of active oxygen scavenging enzyme activity in germinating soybean seedlings under drought stress. By increasing the vitality, the activity of increasing iron oxide content in wheat seedlings is reduced, and the content in seedlings is reduced (Singh and Srivastava, 1999). Pre-treatment inhibits the activity to a certain extent, delays the increase of the content, slows the over-oxidation of membrane lipids in the growth process of young wheat seedlings, and protects the stability of the membrane structure, thereby promoting the growth of young seedlings.

\section{Materials and methods}

\section{Experimental details and treatments}

After the seed undergoes swelling, it enters the stagnant phase of water absorption and the seed enters the internal material activation period. At this stage, the increase of seed respiration, the activation of zymogens and the induction of new enzyme synthesis, proteolysis, synthesis of intracellular macromolecules, etc., require the consumption of a large amount of energy substances. The supply of these energy sources and substances mainly comes from the decomposition of the endosperm or cotyledons. The endosperm is the main storage site for cereals in seeds, of which starch is the main component. Germination of crop seeds is related to many factors, which can be summarized as internal factors and external factors. Internal factors mainly refer to whether the seeds are fully developed and have vitality, whether the seed dormancy is released or not. External factors refer to the external environmental conditions required for the germination of seeds, which play a key role in the normal germination of seeds. Unfavorable external environmental conditions can directly affect the germination of seeds. If these unfavorable factors exceed the normal range of the normal growth and 
development of crop seeds, they will not only affect the germination of crop seeds, but also cause damage and even death of crop growth and development.

\section{Experimental material}

In general, after sowing, the seeds can germinate normally under suitable temperature conditions, and high temperature and low temperature will affect seed germination. The temperature has an effect on the membrane permeability of the cells and the activity of the hydrolase during the germination of the crop. However, the effects of temperature on seed germination mainly show that the three points are: the highest limit temperature, the lowest limit temperature and the most suitable temperature, of which only the most suitable temperature is the most favorable for seed germination. Optimum germination temperature of crops is related to external conditions. For example, the optimum germination temperature of seeds in warm environment is higher than that in cold regions. The $\mathrm{p} \mathrm{H}$ value of the soil has a great influence on the seed germination, and the $\mathrm{p} \mathrm{H}$ value is too high, making the soil strongly alkaline. When seeds are sown in alkaline soil, the seed germination is caused by alkali stress, and strong alkali stress is harmful to the germination of seeds, which can make the seeds burn and cannot germinate normally (Fig. 1). The $\mathrm{p} \mathrm{H}$ value is too low to make the soil acidic, and the seeds of the crop that germinate under the acidic soil will be affected by its physiological metabolism and important osmotic adjustment. Therefore, $\mathrm{pH}$ value is also very important for the germination of crop seeds.

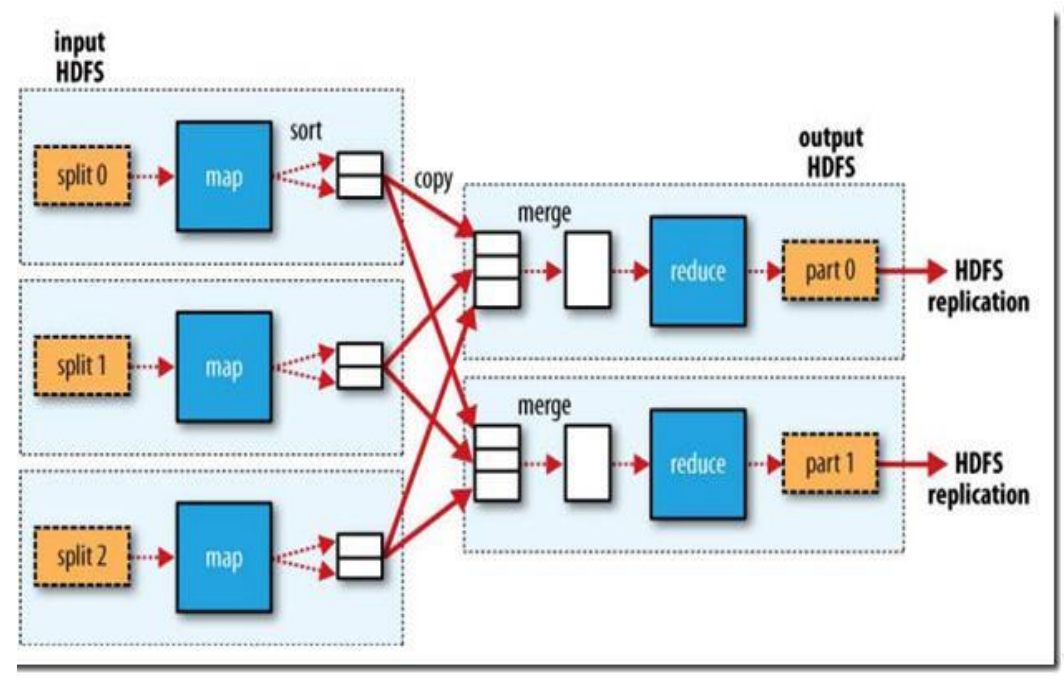

Figure 1. Effect of sodium hydroxide on isozymes of wheat seeds

\section{Treatments}

Embryos generally consist of radicles, hypocotyls, germs, and cotyledons. Under suitable external environmental conditions, seeds can grow into new individual plants as they germinate. Germination of seeds is generally considered to be the initial stage of the plant's entire life activity. Seed germination refers to that the seed breaks through the seed coat under suitable environmental conditions and undergoes a series of physiological metabolic changes. It can be roughly divided into three parts, namely the inhalation, activation and seed germination. The swelling effect is a physical change 
and is an indispensable step for the germination of the seed. At this stage, the seed absorbs a large amount of water, the seed coat gradually softens, and the air permeability is enhanced. The final moisture content of the seed will reach a threshold value. Generally, the seed threshold of different plants is different. This difference is caused by the different components contained within the seed.

\section{Wheat seed hydrolase activity}

At present, heavy metal pollution is a focus of global attention. Due to its characteristics of great harm and difficulty in remediation, many countries attach great importance to it. According to statistics, China's total arable land has been one-fifths contaminated by heavy metals, which has seriously affected the growth and development of crops and the yield and quality of products. One of the stages in the process of plant growth and development during seed germination is most susceptible to external abiotic factors. Once the seeds are germinated, they are subject to heavy metal contamination, which will cause changes in the internal indicators of the seeds. This shows that heavy metals have toxic effects on the germination of plant seeds, but low concentrations of heavy metal ions can stimulate plant growth and development (Fig. 2). The seed germination was promoted. On the contrary, when the concentration was higher, the seed germination was inhibited, and the inhibitory effect was obvious with the increase of the concentration.



Figure 2. Sulfide content standard curve

\section{Mitigative effects of oxidative damage during germination of wheat seeds}

At the same time, it influences various processes of biomechanics such as photosynthesis, respiratory absorption, absorption and transportation of water and nutrition, activity of various enzymes, and transformation, transportation, and accumulation of organic matter. The BOX template in the spatial image processing is to use the pixels to be processed and the eight adjacent pixels around it to average the pixels to remove the pixels that produce mutations. Using this method, the noise of the image can be weakened. It also has some limitations, although the use of BOX template for image processing pays sufficient attention to the use of adjacent pixels, but does not 
pay attention to the impact of pixel orientation on the image, so the effect is not ideal when dealing with complex images. Assume that the input sequence is a non-Gaussian, independent and identically distributed stationary process $(E q .1)$ :

$$
C_{N z}=C_{N x} \cdot \sum_{t} s_{i}^{N}(t)
$$

The constraints are (Eq. 2):

$$
K_{x}(M, N)=\frac{C_{M x}}{\left[C_{N x}\right]^{M / N}}
$$

First describe the data model of an OFDM system (Eq. 3):

$$
K_{1}=L-K_{2}+1, \quad K_{2}=\left[\arg \max _{0 \leq m \leq L-N_{c}} \sum_{i=m}^{m+N_{c}-1}\left|h_{i}\right|^{2}\right]-1
$$

The received signal vector formed at the receiving end is as follows (Eq. 4):

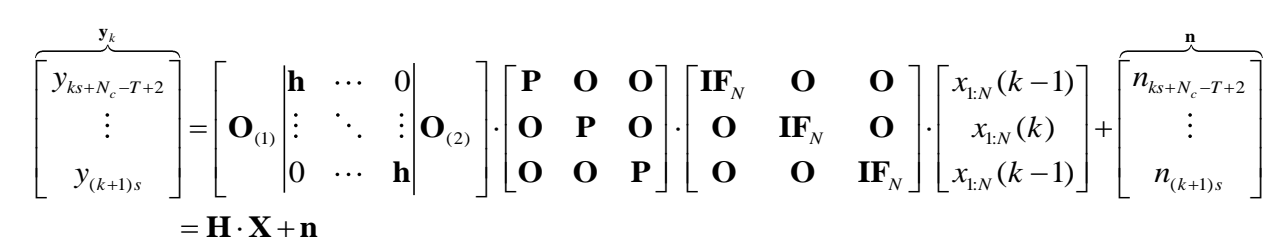

Indicates the process of adding a $\mathrm{CP}(E q .5)$ :

$$
\mathbf{P}=\left[\frac{\mathbf{O} \mid \mathbf{I}_{N c}}{\mathbf{I}_{N}}\right]
$$

Its balanced output is shown as $(E q .6)$ :

$$
\left[\begin{array}{c}
z_{1}(k) \\
\vdots \\
z_{N}(k)
\end{array}\right]=\left[\begin{array}{ccc}
D_{1} & 0 & \cdots \\
0 & \ddots & 0 \\
0 & \cdots & D_{N}
\end{array}\right] \cdot \mathbf{F}_{N} \cdot(\mathbf{Y} \cdot \mathbf{w})
$$

To further reduce complexity (Eq. 7):

$$
\mathbf{Y}=\left[\begin{array}{cccc}
y_{k \cdot s+N_{c}+1} & y_{k \cdot s+N_{c}} & \cdots & y_{k \cdot s+N_{c}-T+2} \\
y_{k \cdot s+N_{c}+2} & y_{k \cdot s+N_{c}+1} & \cdots & y_{k \cdot s+N_{c}-T+3} \\
\vdots & \vdots & \ddots & \vdots \\
y_{(k+1) \cdot s} & y_{(k+1) \cdot s-1} & \cdots & y_{(k+1) \cdot s-T+1}
\end{array}\right]
$$


There are many types of seeds in nature, and seeds are the reproductive organs of seed plants. The seeds produced by different plants will have large differences in morphology and structure. Seed plants include gymnosperms and angiosperms. Most angiosperms are composed of embryos, endosperm and seed coats.

\section{Statistical analysis}

Under the heavy metal stress, the activities of the hydrolase such as amylase and esterase, which are related to seed germination, will change drastically. Amylase, esterase and other hydrolyzing enzymes can hydrolyze macromolecules such as starch and esters into small molecules such as glucose and phosphoric esters and release energy to provide protection for plant seed germination. The stronger the hydrolase activity such as amylase esterase is, the stronger the hydrolyzing power is, and the faster seed germination occurs. Conversely, the weaker the activity is, the weaker the hydrolyzing power is, the energy supply required for seed germination is affected, and the seed germination growth is slow or even inhibited.

\section{Results}

\section{Regulation of iron oxides and sodium hydroxide on the antioxidant system of wheat seeds during germination}

\section{Iron oxides}

From the current research results, it can be seen that higher concentrations of heavy metal ions have a greater impact on the amylase activity of the seed, which severely inhibits the activity of the amylase, thereby impeding the germination of the seed. However, the specific molecular mechanism of heavy metal inhibiting amylase activity is still unclear. In the process of metabolism, it can hydrolyze ester bonds, catalyze the hydrolysis of esters, and provide energy for the germination of plants. In general, the activity of heavy metals on amylase esterase is related to the concentration of heavy metals. High concentrations of heavy metals inhibit the activity of amylase esterase hydrolyzing enzymes, and low concentration treatments have a promoting effect, which corresponds to the morphological indicators of plant seed germination under heavy metal stress (Fig. 3).

Therefore, it is speculated that high concentrations of heavy metals may be harmful to the germination of seeds by inhibiting the activity of these hydrolytic enzymes. Finally, the effect of heavy metals on the seed germination is also reflected in the changes of soluble substances, proline content and antioxidant metabolism of the seeds. Generally speaking, under heavy metal stress, plants can increase the osmotic potential of cells through the accumulation of their own organic substances and maintain the normal metabolism of cells. The soluble substances not only participate in the important osmotic adjustment in the plant, but also provide the energy source for the plants and provide the starting material for the synthesis of other organic compounds in the plant.

\section{Sodium hydroxide}

Generally speaking, adverse stress has an adverse effect on the growth and development of plants, but under adverse circumstances, the plant's life activities itself will produce a special law to adapt to adversity stress, so that it can better adapt to the 
environment. Under stress, the content of stored substances in the seed of the plant will change greatly, generally increasing the content of organic matter such as soluble sugar and soluble protein in the plant. Soluble sugars are involved in the osmotic adjustment of plant cells. The increase of soluble sugar content under stress can increase the osmotic potential of cells and maintain the normal metabolism of cells. The increase of soluble protein content will increase the number of functional proteins in the cells, allowing the plants to better adapt to adversity stress and survive in adverse environments. Therefore, the changes of soluble sugar and soluble protein content can reflect the growth status of the seeds, which is beneficial to improve plant stress resistance (Table 1). In addition, proline is an important substance involved in the seed germination and osmotic adjustment. Under the stress of adversity, the content in the germinating seeds increases rapidly, and its accumulation helps the plants to adapt to adversity, so the content can reflect.

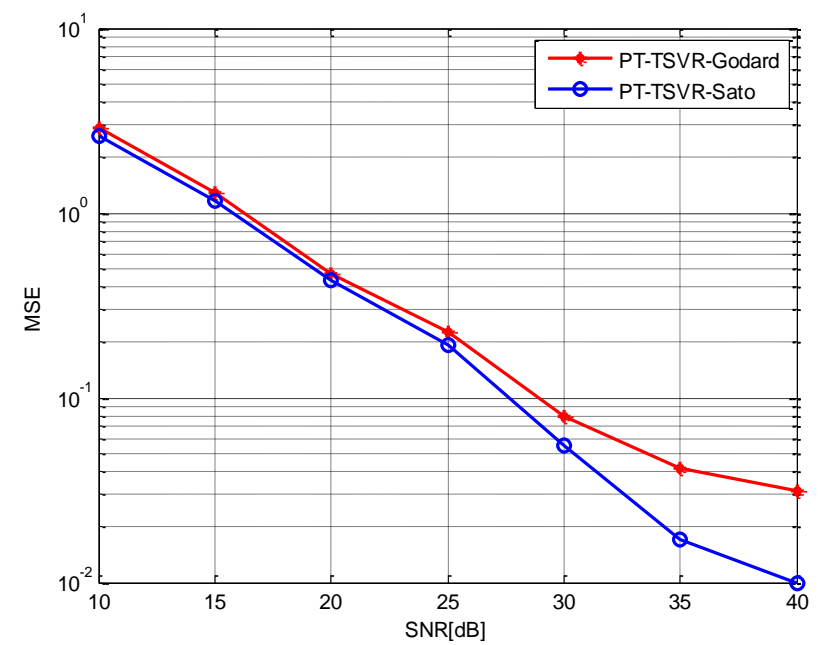

Figure 3. Protein content in wheat seed germination

Table 1. Each category classification result data table

\begin{tabular}{c|c|c}
\hline Recall & Precision rate & Average assessment \\
\hline $72.779 \%$ & $79.091 \%$ & $75.935 \%$ \\
$69.584 \%$ & $81.954 \%$ & $75.769 \%$ \\
$70.895 \%$ & $81.285 \%$ & $76.090 \%$ \\
$71.684 \%$ & $80.059 \%$ & $75.871 \%$ \\
$70.597 \%$ & $79.761 \%$ & $75.179 \%$ \\
$64.119 \%$ & $87.931 \%$ & $76.025 \%$ \\
$71.333 \%$ & $88.000 \%$ & $79.667 \%$ \\
$81.519 \%$ & $65.692 \%$ & $73.606 \%$ \\
$59.446 \%$ & $74.441 \%$ & $66.944 \%$ \\
$87.329 \%$ & $87.671 \%$ & $87.500 \%$ \\
$80.353 \%$ & $86.246 \%$ & $63.300 \%$ \\
$75.671 \%$ & $79.429 \%$ & $77.550 \%$ \\
$79.463 \%$ & $81.750 \%$ & $80.607 \%$ \\
$73.915 \%$ & $84.825 \%$ & $79.370 \%$ \\
$84.407 \%$ & $66.922 \%$ & $75.665 \%$ \\
\hline
\end{tabular}




\section{Discussion}

Protein is a macromolecular substance rich in cells, and it is one of the key basic substances in the structure and function of crop seeds. Heavy metal stress can increase the soluble protein content of crop seeds, and then increase the number of functional proteins, which is conducive to the normal metabolic activity of crop seed cells, and increase the resistance of plants to heavy metals. Proline is involved in the key osmotic adjustment of crops. The change of content can reflect the strength of crop resistance. Therefore, the content of proline is used as a physiological indicator of crop stress resistance. Under normal circumstances, the content of proline in crops is relatively low, and the increase of content may be one of the mechanisms of resistance of plants. Smirnoff pointed out that the praline of crops can remove active oxygen accumulated under adverse conditions.

Seed germination is constrained by both internal and external factors, among which internal factors include dormancy and immature seeds, and seed coat restriction and other external factors including light, oxygen, and chemical substances. The minerals in the soil, salt, are crucial for the germination of seeds. However, in recent years, the nutritional structure of the soil has changed due to the destruction of the environment, the lack of water resources, and the abuse of chemical fertilizers. Soil salinization becomes a resource issue and an ecological problem that affects the entire world. The seed germination process starts because photosynthesis cannot be performed. The energy and materials required are supplied by the nutrients stored in the organs. When nutrients are mobilized, they will be converted into easily transportable forms, transported to the most active and fastest growing areas of metabolism, providing energy and carbon skeletons and other substances for a range of physiological activities during germination. Starch and fat are the most common and major storage material for wheat seeds. Although hydrolyzing enzymes such as amylases, etc. are not necessarily required in the germination of wheat seeds, the growth and development of seedlings depends on the activation of storage substances in the endosperm.

In this section, we investigated the changes of amylase isoenzyme activity during the seed germination of wheat under salt stress. Salt stress and aluminum stress have significant toxic effects on the germination of wheat seeds, and they are expressed in the length of radicles, embryos, and the number of radicles and germination rate. Among them, the effect of aluminum stress on radicle growth was particularly obvious. After germination and treatment of wheat seeds, their germination status was significantly relieved, and radicle germination became normal.

Acknowledgements. This work was supported by the National Natural Science Foundation of China (No. 31271627) and Henan Provincial Research Foundation for Science and Technological Breakthroughs, China (Grant No. 152102110106).

\section{REFERENCES}

[1] Aoyama, I., Okamura, H. (2010): Interactive toxic effect and bioconcentration between cadmium and chromium using continuous algal culture. - Environmental Toxicology 8: 55-269.

[2] Awad, F. (2000): Mobilization of heavy metals from contaminated calcareous soils by plant borne, microbial and synthetic chelators and their uptake by wheat plants. - Journal of Plant Nutrition 23: 1847-1855. 
[3] Awad, F., Römheld, V. (2000): Mobilization of heavy metals from a contaminated calcareous soil by plant borne and synthetic chelators and its uptake by wheat plants. Journal of Plant Nutrition 23: 1847-1855.

[4] Bauer, M., Blodau, C. (2006): Mobilization of arsenic by dissolved organic matter from iron oxides, soils and sediments. - Science of the Total Environment 354: 179-190.

[5] Bose, S., Bhattacharyya, A. K. (2008): Heavy metal accumulation in wheat plant grown in soil amended with industrial sludge. - Chemosphere 70: 1264-1272.

[6] Bowell, R. J. (1994): Sorption of arsenic by iron oxides and oxyhydroxides in soils. Applied Geochemistry 9: 279-286.

[7] Cenci, G., Morozzi, G. (1977): Evaluation of the toxic effect of cd2+ and cd 42- ions on the growth of mixed microbial population of activated sludges. - Science of the Total Environment 7: 131-143.

[8] Clausen, L., Fabricius, I. (2001): Atrazine, isoproturon, mecoprop and bentazone adsorption onto iron oxides. - Journal of Environmental Quality 30: 858.

[9] Debetto, P., Luciani, S. (1988): Toxic effect of chromium on cellular metabolism. Science of the Total Environment 71: 365-377.

[10] Hagenbuch, I. M., Pinckney, J. L. (2012): Toxic effect of the combined antibiotics ciprofloxacin, lincomycin, and tylosin on two species of marine diatoms. - Water Research 46: 5028-5036.

[11] Hanesch, M. (2010): Raman spectroscopy of iron oxides and (oxy) hydroxides at low laser power and possible applications in environmental magnetic studies. - Geophysical Journal International 177: 941-948.

[12] Lanzky, P. F., Halling-Sã,Rensen, B. (1997). The toxic effect of the antibiotic metronidazole on aquatic organisms. - Chemosphere 35: 2553-61.

[13] Källqvist, T., Meadows, B. S. (1978): The toxic effect of copper on algae and rotifers from a soda lake. - Water Research 12: 771-775.

[14] Mishra, K. B., Gopal, R. (2008): Detection of nickel induced stress using laser-induced fluorescence signatures from leaves of wheat seedlings. - International Journal of Remote Sensing 29: 157-173.

[15] Mittal, S. K., Ratra, R. K. (2000): Toxic effect of metal ions on biochemical oxygen demand. - Water Research 34: 147-152.

[16] Muslu, A., Ergün, N. (2013): Effects of copper and chromium and high temperature on growth, proline and protein content in wheat seedlings. - Bangladesh Journal of Botany 42: 105-111.

[17] Okamura, H., Aoyama, I. (2010): Interactive toxic effect and distribution of heavy metals in phytoplankton. - Environmental Toxicology 9: 7-15.

[18] Prado, A. G. S., Airoldi, C. (2002): The toxic effect on soil microbial activity caused by the free or immobilized pesticide diuron. - Thermochimica Acta 394: 155-162.

[19] Schulze, D. G. (1984): The influence of aluminium on iron oxides: x. properties of alsubstituted goethites. - Clay Minerals 19: 521-539.

[20] Schwertmann, H. C. U., Cornell, R. M. (2000): Iron oxides in the laboratory: preparation and characterization. - Clay Minerals 27: 393-393.

[21] Singh, A., Srivastava, V. K. (1999): Toxic effect of synthetic pyrethroid permethrin on the enzyme system of the freshwater fish Channa striatus. - Chemosphere 39: 1951-1956.

[22] Tkachenko, A. G. (2004): Mechanisms of protective functions of escherichia coli, polyamines against toxic effect of paraquat, which causes superoxide stress. Biochemistry 69: 188-194. 